\title{
Combined PET and Biopsy Evidence of Marrow Involvement Improves Prognostic Prediction in Diffuse Large B-Cell Lymphoma
}

\author{
Juliano J. Cerci ${ }^{1}$, Tamás Györke ${ }^{2,3}$, Stefano Fanti ${ }^{4}$, Diana Paez ${ }^{5}$, José Cláudio Meneghetti ${ }^{6}$, Francisca Redondo ${ }^{7}$, \\ Monica Celli $^{4}$, Chirayu Auewarakul ${ }^{8}$, Venkatesh Rangarajan ${ }^{9}$, Sumeet Gujral ${ }^{9}$, Charity Gorospe ${ }^{10}$, Maejoy V. Campo ${ }^{10}$, \\ June-Key Chung ${ }^{11}$, Tim P. Morris ${ }^{12}$, Maurizio Dondi ${ }^{4}$, and Robert Carr ${ }^{13}$ on behalf of the IAEA Lymphoma Study Group \\ ${ }^{1}$ Quanta Diagnóstico e Terapia, Curitiba, Brazil; ${ }^{2}$ ScanoMed Medical Diagnostic Ltd., Budapest, Hungary; ${ }^{3}$ Department of Nuclear \\ Medicine, Semmelweis University, Budapest, Hungary; ${ }^{4}$ Policlinico S. Orsola Malpighi, Universita de Bologna, Bologna, Italy; \\ ${ }^{5}$ Division of Human Health, Department of Nuclear Sciences and Application, International Atomic Energy Agency (IAEA), Vienna, \\ Austria; ${ }^{6}$ Hospital de Clinicas, Universidade de São Paulo, São Paulo, Brazil; ${ }^{7}$ Oncologic Clinic, Fundación Arturo Lopez Perez, \\ Santiago, Chile; ${ }^{8}$ Chulabhorn Cancer Centre and Faculty of Medicine Siriraj Hospital, Bangkok, Thailand; ${ }^{9}$ Departments of Nuclear \\ Medicine and Pathology, Tata Memorial Hospital, Mumbai, India; ${ }^{10}$ St. Luke's Medical Center, Quezon City, Manila, Philippines; \\ ${ }^{11}$ Seoul National University Hospital, Seoul, South Korea; ${ }^{12}$ MRC Clinical Trials Unit at UCL, Institute of Clinical Trials and \\ Methodology, London, United Kingdom; and ${ }^{13}$ Department of Haematology, Guy's and St Thomas' Hospital, London, United Kingdom
}

Bone marrow is an important extranodal site in diffuse large B-cell lymphoma (DLBCL), and marrow histology has been incorporated into the new National Comprehensive Cancer Network international prognostic index. Marrow involvement demonstrated histologically confers poor prognosis but is identified by staging PET in more cases. How information from staging PET and biopsy should be combined to optimize outcome prediction remains unclear. Methods: The International Atomic Energy Agency sponsored a prospective international cohort study to better define the use of PET in DLBCL. As a planned subsidiary analysis, we examined the interplay of marrow involvement identified by PET and biopsy on clinical outcomes. Results: Eight countries contributed 327 cases with a median follow-up of 35 mo. The 2-y outcomes of cases with no evidence of marrow involvement $(n=231)$ were $81 \%$ (95\% confidence interval [Cl], 76\%-86\%) for event-free survival (EFS) and $88 \%(83 \%-91 \%)$ for overall survival (OS); cases identified only on PET ( $n=61), 81 \%$ (69\%-89\%) for EFS and $88 \%(77 \%-94 \%)$ for OS; cases indentified only on biopsy $(n=10), 80 \%$ (41\%-95\%) for EFS and $100 \%$ for OS; or cases identified by both PET and biopsy $(n=25)$, $45 \%(25 \%-64 \%)$ for EFS and 55\% (32\%-73\%) for OS. The hazard ratios for PET-negative/biopsy-negative cases versus PET-positive/ biopsy-positive cases were $2.67(95 \% \mathrm{Cl}, 1.48-4.79)$ for EFS and 3.94 (1.93-8.06) for OS. Conclusion: This large study demonstrates that positive iliac crest biopsy histology only confers poor prognosis for patients who also have abnormal marrow ${ }^{18}$ F-FDG uptake identified on the staging PET scan. Abnormal ${ }^{18} \mathrm{~F}-\mathrm{FDG}$ uptake in marrow, when iliac crest biopsy histology is normal, has no adverse effect on outcomes.

Key Words: positron emission tomography; diffuse large B-cell lymphoma; bone marrow; International Prognostic Index

J Nucl Med 2014; 55:1591-1597

DOI: 10.2967/jnumed.113.134486

Received May 22, 2014; revision accepted Aug. 1, 2014.

For correspondence or reprints contact: Juliano J. Cerci, Division of PET/CT, Quanta-Diagnóstico Nuclear, R. Almirante Tamandaré, 1000, CEP 80045-170, Curitiba, Brazil.

E-mail: cercijuliano@hotmail.com

Published online Sep. 11, 2014

COPYRIGHT (C 2014 by the Society of Nuclear Medicine and Molecular Imaging, Inc.
D ment and tra design in diffuse large B-cell lymphoma (DLBCL). The international prognostic index (IPI), which includes age, Ann Arbor stage, Eastern Cooperative Oncology Group performance status, extranodal disease, and abnormal lactate dehydrogenase (LDH), has been the gold standard for $20 \mathrm{y}$ (1). In the era of rituximab immunochemotherapy, with attendant improved survivals, the IPI is less able to discriminate higher risk patients (2).

The recently published National Comprehensive Cancer Networkrevised IPI achieved significant improvement in discrimination between low- and high-risk subgroups, based on overall survival (OS), for rituximab-treated patients (3). This enhancement is achieved by adding 3 age categories and 2 strata of elevated LDH and replacing the number of extranodal sites with involvement of specific organs, namely bone marrow, central nervous system, liver/ gastrointestinal tract, or lung. Marrow involvement is defined as positive iliac crest histology, shown to confer poor prognosis in a large retrospective database study (4).

During the past 2 decades, PET has become a pivotal component of lymphoma staging through its ability to identify tumor cells in anatomically normal lymph nodes and in extranodal sites (5). Early PET studies drew attention to the fact that marrow involvement in DLBCL was much more frequently metastatic, with isolated areas of focal ${ }^{18} \mathrm{~F}-\mathrm{FDG}$ uptake in one or many sites throughout the medullary skeleton, and less frequently identified as diffusely increased ${ }^{18}$ F-FDG uptake (6). That PET consistently identifies marrow disease in more patients than biopsy is now accepted (7-9). More recent studies have attempted to establish the prognostic significance of marrow disease when identified either by PET or by routine, untargeted iliac crest biopsy. Two such studies reported that positive marrow histology conferred a worse prognosis, whereas the third found that marrow involvement identified by PET, but not biopsy, was the only independent predictor for poor outcome (10-12).

A recent systematic review of PET/CT for detecting bone marrow involvement found strong evidence for its accuracy and complementary role in detecting marrow disease in newly diagnosed DLBCL. 
At the same time, the authors highlighted the need for further studies to determine the relative role of PET and bone marrow biopsy (BMB) in determining prognosis (13).

We have recently completed a prospective multinational cohort study, sponsored by the International Atomic Energy Agency (IAEA), to investigate the application of PET for risk assessment of DLBCL. As a planned subsidiary analysis, we investigated marrow involvement identified by staging PET and routine iliac crest biopsy for predicting treatment outcomes and to inform international practice. Our hypothesis, based on recent literature (11) and the most recent international consensus guidance on the use of PET/CT for lymphoma staging (14), was that $\mathrm{BMB}$ is no longer necessary in the era of routine staging by PET.

\section{MATERIALS AND METHODS}

Major cancer centers in 8 countries (in Sâo Paulo, Brazil; Santiago, Chile; Budapest and Debrecen, Hungary; Mumbai, India; Bologna, Italy; Seoul, South Korea; Manila, Philippines; Bangkok, Thailand) from 5 United Nations-defined regions participated in the study. Recruitment commenced in 2008 through September 2011.

\section{Eligibility Criteria}

Participating clinicians recruited sequential cases of DLBCL, with patients aged $16 \mathrm{y}$ or older, provided that informed consent could be obtained before chemotherapy. Exclusions were central nervous system involvement, cancer within preceding $5 \mathrm{y}$, and steroid therapy before staging scan.

\section{Study Protocol}

The protocol was developed by the study lead clinicians from each country during 2 investigator meetings. Diagnosis of DLBCL was based on histology with standard immunohistochemistry to include, at minimum, CD20, CD3, and either Ki-67 or MiB1 (15). All patients were staged by PET/CT (6 centers) or PET and CT separately (Italy and Brazil) and by iliac crest biopsy. Recommended treatment was 6 cycles of R-CHOP (rituximab with cyclophosphamide, adriamycin, vincristine, prednisolone) at 21-d intervals. To accommodate local practice, R-CHOP up to 8 cycles was permitted. Omission of rituximab was allowed in recognition that some eligible patients might otherwise be excluded for financial reasons. All patients underwent PET scans after 2-3 cycles of chemotherapy and 4-8 wk after all chemotherapy as part of the study design to assess response at nodal and extranodal sites. Treatment modification in response to the midtreatment PET scan was not permitted, except in cases with confirmed disease progression.

Identification of Marrow Disease by PET. Marrow involvement on the staging PET or PET/CT (together referred to as PET throughout the manuscript) scan was classified as focal or diffuse as previously described (6). Focal was defined as 1 or more circumscribed areas of high ${ }^{18} \mathrm{~F}-\mathrm{FDG}$ uptake within the skeleton and diffuse as uniform increased ${ }^{18} \mathrm{~F}-\mathrm{FDG}$ uptake throughout the bone marrow space. Diffuse ${ }^{18}$ F-FDG uptake was not considered to represent lymphoma involvement unless supported by positive biopsy histology.

The criteria for focal ${ }^{18} \mathrm{~F}-\mathrm{FDG}$ uptake in bone marrow to be classified as due to DLBCL were as previously reported by others $(11,16):{ }^{18} \mathrm{~F}-$ FDG uptake to be greater than the intensity of uptake in normal liver, with no anatomic changes to suggest alternative benign bone pathology. Bone or marrow involvement by spread from a contiguous nonskeletal site was excluded. Patients were considered to have bone marrow involvement by lymphoma if they had either histologic DLBCL in the marrow biopsy or focal PET-positive $(\mathrm{PET}+)$ marrow involvement irrespective of iliac crest biopsy histology.

\section{Histologic Identification of Marrow Disease}

All cases had untargeted unilateral iliac crest biopsy. BMB histology immunohistochemistry was required to include CD20, CD3, and CD10 for accurate detection of low-level marrow involvement (15).

\section{Classification of Events}

Study events were relapse after complete remission, death from any cause, treatment escalation for progressive disease while on treatment, and disease progression or failure to achieve complete remission at the end of chemotherapy based on the revised response criteria for lymphoma (17). The protocol required suspected residual disease to be confirmed by biopsy.

\section{Data Quality Assurance}

Each patient record was reviewed with the country chief investigator during the final collaborator meeting for consistency and accuracy.

\section{Research Regulation and Data Protection}

Each country gained research ethics approval for the study protocol and patient information from the appropriate national or local Ethics Review Board. Some countries imposed unusually strict recruitment criteria, which limited recruitment in some larger centers. Fully informed consent was an inclusion criteria for recruitment. Signed consent forms are kept by the local investigators. To ensure confidentiality while sharing data internationally, cases were assigned a numeric code, and only 2 identifiers for data validation, initials and date of birth, were recorded in the central database (18).

\section{Statistical Methods}

Follow-up was continued until $75 \%$ of patients had reached 2 y or died. Cases lost to follow-up were censored at date of last known disease status. Survival was estimated using Kaplan-Meier methods. Event-free survivals (EFS) and OSs are reported. Survival times are all reported at $2 \mathrm{y}$, using date of first chemotherapy as the origin. The

TABLE 1

Patient Characteristics

\begin{tabular}{lcc}
\hline \multicolumn{1}{c}{ Characteristic } & $n$ & Percentage \\
\hline Male sex & 173 & $53 \%$ \\
\hline IPI & 135 & \\
\hline $0-1$ & 81 & $41 \%$ \\
\hline 2 & 68 & $25 \%$ \\
\hline 3 & 43 & $21 \%$ \\
\hline $4+$ & & $13 \%$ \\
\hline Stage & 22 & \\
\hline I & 95 & $6 \%$ \\
\hline II & 64 & $29 \%$ \\
\hline III & 146 & $20 \%$ \\
\hline IV & & $45 \%$ \\
\hline Performance status & 276 & \\
\hline $0-1$ & 36 & $84 \%$ \\
\hline 2 & 9 & $11 \%$ \\
\hline 3 & 6 & $3 \%$ \\
\hline 4 & 166 & $2 \%$ \\
\hline LDH $>$ normal & 95 & $51 \%$ \\
\hline Extranodal sites $\geq 2$ & & $29 \%$ \\
\hline Median age was 55 y (quartiles, & 44,63 y). \\
\hline
\end{tabular}


TABLE 2

Marrow Status Pretreatment Defined by Staging PET and BMB

\begin{tabular}{lcccc}
\hline \multicolumn{1}{c}{ PET } & Total patients & BMB- & BMB+ \\
\hline Negative & 241 & 231 & 10 & 10 \\
Positive, focal & 68 & 47 & 21 & 4 \\
Positive, diffuse & 18 & $14^{*}$ & 38 \\
Total & 327 & 292 & 4
\end{tabular}

*These 14 cases were classified as not having marrow involvement by lymphoma.

prognostic impact of bone marrow involvement, as identified by PET or biopsy, is estimated from a Cox proportional hazards model and hazard ratios (HRs) reported (19).

Multivariate analysis was undertaken to investigate whether marrow involvement identified by PET alone (focal or diffuse combined), by biopsy alone, or by both PET and biopsy was an independent predictor of outcome. The model included the component clinical indicators from the IPI (without National Comprehensive Cancer Network modifications), that is, age ( $>60$ ), stage (III/IV), performance status ( $\geq 2), \mathrm{LDH}$ greater than normal, and extranodal disease in 2 sites or more. The model also includes use or omission of rituximab from therapy. Analyses and graphs were produced using Stata 12 (StataCorp.).

\section{RESULTS}

Eight countries recruited 327 cases. The number of cases from each country was 61 from Brazil, 47 from Chile, 65 from Hungary, 32 from India, 49 from Italy, 9 from South Korea, 20 from Philippines, and 44 from Thailand. Patient characteristics are listed in Table 1 . Median age was $55 \mathrm{y}$, and $35 \%$ of patients were older than $60 \mathrm{y} ; 280(86 \%)$ patients received rituximab.

\section{Identification of Marrow Disease}

Staging was by PET/CT $(n=217)$ or separate PET and CT (Italy, Brazil; $n=110)$. Eighty-two of the 327 cases $(25 \%)$ were considered to have lymphoma in marrow at diagnosis; 47 of 82 (57\%) were identified by PET alone, $10(12 \%)$ by BMB alone, and $25(30 \%)$ by both PET and BMB (Table 2$)$.

Of 241 judged to have normal marrow on PET, 10 (4\%) had positive biopsies. Of these, 2 had deposits of indolent lymphoma, 6 had a low-level infiltrate $(<10 \%)$ of DLBCL cells, and only 2 had a DLBCL infiltrate of more than $10 \%$ nucleated cells in the iliac crest sample. Of 68 focal PET + cases, 21 (31\%) had lymphoma detected by biopsy. Of 18 with diffuse ${ }^{18} \mathrm{~F}-\mathrm{FDG}$ uptake throughout skeletal marrow including the pelvis, only 4 had histologic evidence of marrow disease on iliac crest biopsy (Table 2).

\section{Marrow Disease and Stage}

In the whole cohort, 146 (45\%) of the cases were stage IV. PET identified marrow involvement not identified by marrow biopsy, which upstaged 23 patients to stage IV: 1 patient from I to IV, 11 from II to IV, and 11 from III to IV; all had focal marrow ${ }^{18} \mathrm{~F}-\mathrm{FDG}$ uptake. No patients had their stage increased by iliac crest biopsy.

The distribution of extranodal disease in the 146 stage IV patients is shown in Table 3.

\section{Marrow Disease and Survival}

Median follow-up was 35 mo. Thirty-five cases had marrow involvement identified by iliac crest histology: 2-y EFS for BMBpositive $(\mathrm{BMB}+)$ cases, $56 \%(95 \%$ confidence interval [CI], 37\%$71 \%$ ), and for BMB-negative (BMB-) cases, 81\% (76\%-85\%); and OS for $\mathrm{BMB}+, 68 \%(49 \%-82 \%)$, and BMB-, $88 \%(83 \%-91 \%)$. The HR for progression-free survival was 2.22 (1.31\%-3.27) (Fig. 1).

Classified by both PET and biopsy status (Fig. 2), there were 231 patients who had neither increased ${ }^{18} \mathrm{~F}-\mathrm{FDG}$ uptake in marrow on PET nor histologic evidence of lymphoma infiltration on biopsy (PET-/BMB-) and had a 2-y EFS of $81 \%$ (95\% CI, 76\%-86\%) and an OS of $88 \%(83 \%-91 \%) ; 61$ patients who had ${ }^{18} \mathrm{~F}-\mathrm{FDG}$ uptake on PET but a negative biopsy (PET+/BMB-) and an EFS of $81 \%(69 \%-89 \%)$ and an OS of 88\% (77\%-94\%); and 10 patients who had marrow involvement identified only by biopsy histology

TABLE 3

Distribution of Extranodal Disease in Stage IV Patients

\begin{tabular}{|c|c|c|c|}
\hline \multirow[b]{2}{*}{ Extranodal sites } & \multicolumn{3}{|c|}{ No. of patients } \\
\hline & Total & Single extranodal organ involved & $\geq 2$ extranodal organs involved \\
\hline Bone marrow only* & 23 & 23 & - \\
\hline Soft tissue (non-bone marrow) only & 64 & 28 & 36 \\
\hline Soft tissue and bone marrow ${ }^{\dagger}$ & 59 & - & 59 \\
\hline Total & 146 & 51 & 95 \\
\hline \multicolumn{4}{|c|}{$\begin{array}{l}{ }^{*} \text { All cases for which bone marrow was identified as the only extranodal organ involved had marrow disease identified by PET but not by } \\
\text { opsy. } \\
{ }^{\dagger} \text { All cases for which marrow biopsy histology was positive had at least one other, nonmarrow extranodal organ involved-that is, had at } \\
\text { ast } 2 \text { extranodal organs involved by lymphoma. }\end{array}$} \\
\hline
\end{tabular}




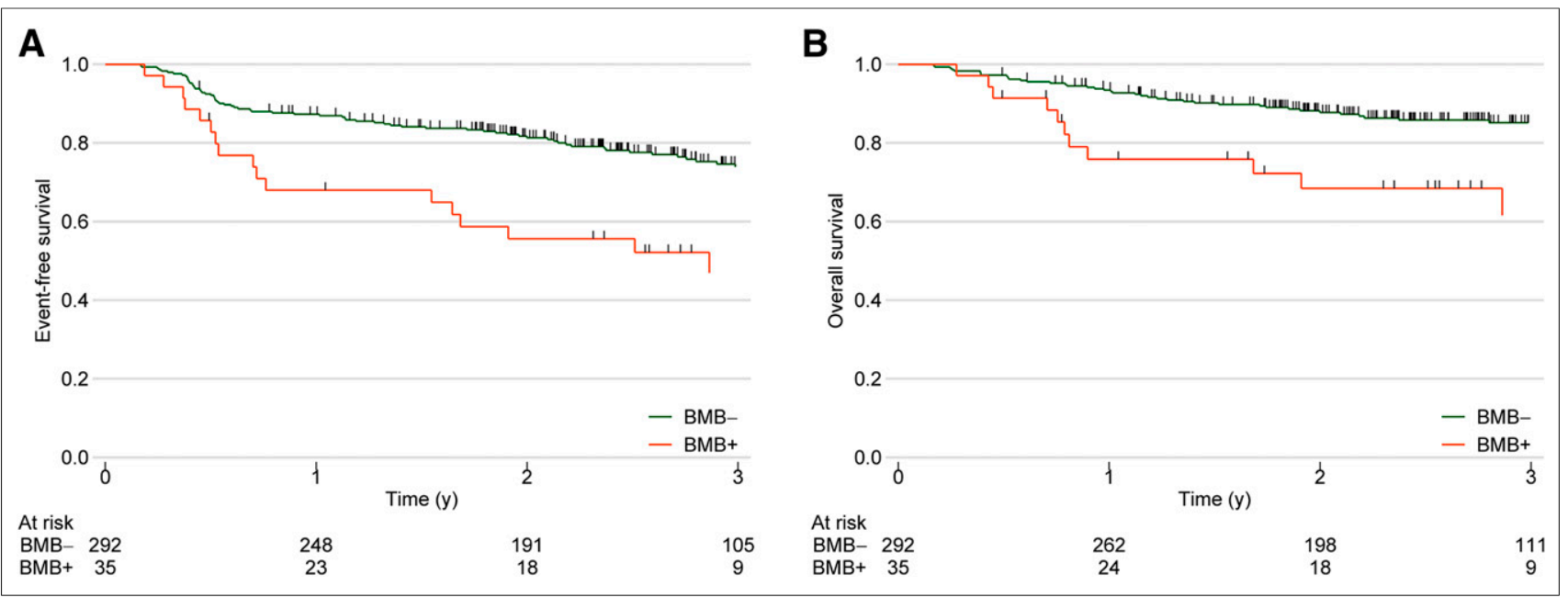

FIGURE 1. EFS (A) and OS (B) of all cases, categorized by marrow biopsy histology (BMB) status. Number of cases at risk in analysis is shown below each time point.

(PET-/BMB +), representing 29\% of histology positive cases, with an EFS of $80 \%(41 \%-95 \%)$ and an OS of $100 \%$. There was no significant difference in EFS or OS between cases with no evidence of marrow disease and those with marrow involvement identified by either PET or BMB alone.

In contrast, 25 patients with marrow involvement identified by both PET and iliac crest biopsy $(\mathrm{PET}+/ \mathrm{BMB}+)$ had significantly inferior 2-y outcomes, with an EFS of $45 \%$ (95\% CI, 25\%-64\%) and an OS of $55 \%(32 \%-73 \%)$. HRs for double-positive (PET+ $/ \mathrm{BMB}+$ ) versus uninvolved (PET-/BMB-) bone marrow were 2.67 (1.48-4.79) for EFS and 3.94 (1.93-8.06) for OS (Fig. 2).

The most frequent pattern of marrow involvement by DLBCL on staging PET is as multiple circumscribed, focal ${ }^{18} \mathrm{~F}-\mathrm{FDG}$-avid deposits. Because less than a third of focal PET-positive cases had disease detected histologically by iliac crest biopsy in this and other series, we explored the prognostic effect of focal disease and whether prognosis was influenced by the presence or absence of positive histology. Of the 68 cases in our series, 47 had negative biopsies (focal PET+/BMB-), EFS of 78\% (95\% CI, 63\%-88\%), and OS of $87 \%$ (73\%-94\%); and 21 had positive biopsies (focal $\mathrm{PET}+\mathrm{BMB}+)$, EFS of $46 \%(24 \%-65 \%)$, and OS of $57 \%(31 \%-$ $76 \%)$. HRs were 2.47 (1.07-5.51) for EFS and 3.03 (1.10-8.40) for OS (Fig. 3).

Multivariate analysis was performed to explore the relative influence on survival of the IPI component clinical indicators, rituximab treatment, and marrow disease detected by only PET or biopsy or both PET and biopsy (Table 4). When adjusted for each of these covariates, the effect of marrow involvement detected by both PET and BMB together had a significant independent adverse impact on OS, with an HR of 2.26 (95\% CI, 1.02-5.03) $(P=0.05)$. Marrow involvement identified by marrow biopsy histology alone had no impact on OS, with an HR of $0.49(0.07-3.68)(P=0.49)$. Whether rituxmab was part of therapy had no effect $(P=0.90)$.

\section{DISCUSSION}

The purpose of this analysis of the IAEA Lymphoma Study data was to better define the relative importance of staging PET,
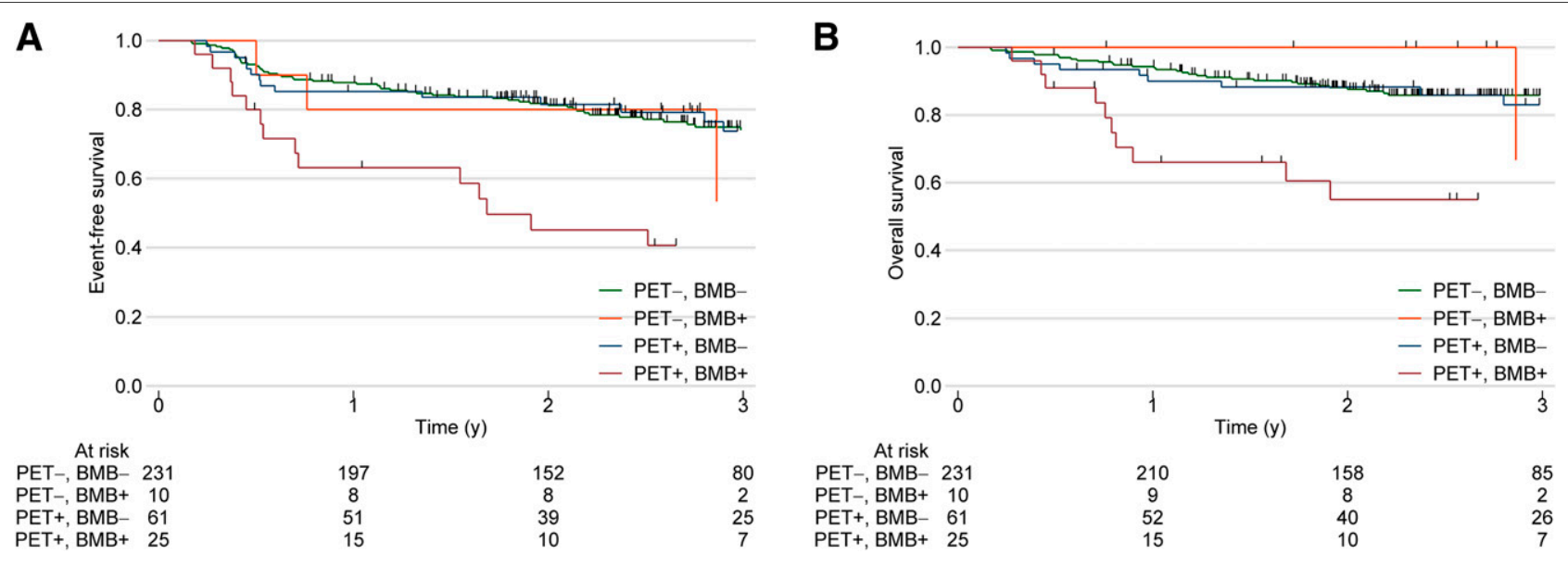

FIGURE 2. Marrow status categorized by both staging PET and marrow biopsy histology (BMB). EFS (A) and OS (B) of all 327 cases. BMB $+=$ iliac crest biopsy shows histologic evidence of lymphoma; BMB- = iliac crest biopsy shows normal marrow histology; PET $+=$ abnormal ${ }^{18}$ F-FDG uptake in marrow, either focal or diffuse pattern; PET- = normal marrow with no increased ${ }^{18} \mathrm{~F}-\mathrm{FDG}$ uptake. Number of cases at risk in analysis is shown below each time point. 


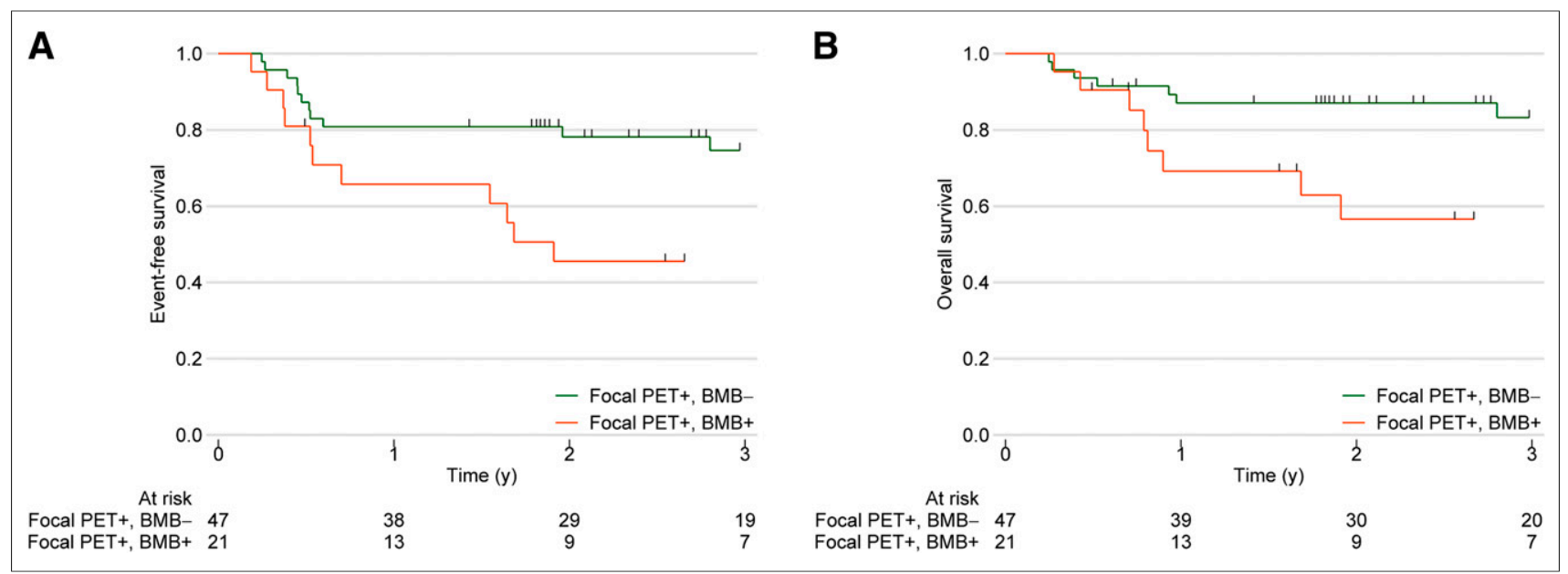

FIGURE 3. Subgroup analysis of focal PET+ cases, categorized by marrow histology. EFS (A) and OS (B) of 68 cases with focal ${ }^{18} \mathrm{~F}-\mathrm{FDG}$ uptake (focal PET+) on staging PET. Number of cases at risk in analysis is shown below each time point.

compared with routine iliac crest biopsy, for establishing the prognostic impact of marrow involvement in DLBCL. The analysis was based on cases of DLBCL from 8 countries in 5 geographic regions treated to a common protocol.

The IAEA cohort of 327 patients is as large as all 3 previous studies, which examined the prognosis of marrow involvement (10-12). In the cohort, $25 \%$ were judged to have marrow involvement by lymphoma; $88 \%$ of these were identified by PET, $42 \%$ by histology (Table 2). These proportions are similar to a recent single-center U.K. study (11). Cases with positive marrow histology had inferior outcomes, compared with those with normal biopsies, as reported by others (10-12). However, when information from staging PET and biopsy were considered together, it was apparent that histologypositive marrow disease did not uniformly predict for poor outcome.

When both PET and biopsy data were integrated, there were 3 separate diagnostic categories of marrow involvement (Fig. 2): marrow infiltration detected by histology with negative marrow on PET (PET-/BMB +), abnormal ${ }^{18}$ F-FDG uptake on PET with negative histology (PET+/BMB-), and abnormal ${ }^{18} \mathrm{~F}-\mathrm{FDG}$ uptake in marrow with positive histology $(\mathrm{PET}+/ \mathrm{BMB}+)$. Cases with marrow involvement identified by a single diagnostic modality had a 2 -y EFS of $80 \%$ and $81 \%$, respectively, similar to patients with no evidence of marrow disease irrespective of stage, with an EFS of $81 \%$. Only cases with marrow involvement identified by both PET and biopsy histology had significantly inferior survivals, with an EFS of $45 \%$, OS of 55\%, and HRs of 2.67 and 3.94, respectively, compared with patients with no marrow involvement.

Multivariate analysis demonstrated that the inferior survival of $\mathrm{PET}+/ \mathrm{BMB}+$ cases was independent of the IPI and its components and independent of marrow involvement detected by either PET or biopsy histology alone. In this model, positive marrow histology did not by itself predict for poor EFS or OS.

The highly significant adverse effect on prognosis of marrow disease identified by both PET and histology, in contrast to the lack of effect when identified by only a single modality, needs further consideration.

Twenty-nine percent (10/35) of cases with positive marrow histology had normal marrow on staging PET and survivals that were not inferior to cases without extranodal spread to marrow. It has been previously reported that a marrow infiltrate of up to $10 \%$ DLBCL cells, or by indolent lymphoma cells, may not accumulate ${ }^{18} \mathrm{~F}-\mathrm{FDG}$ above the metabolic activity of normal

TABLE 4

Multivariable Analysis of Clinical Indicators and Marrow Status by PET/BMB on Survival

\begin{tabular}{|c|c|c|c|c|}
\hline \multirow[b]{2}{*}{ Variable } & \multicolumn{2}{|l|}{ Os } & \multicolumn{2}{|l|}{ EFS } \\
\hline & $\mathrm{HR}$ & $P$ & $\mathrm{HR}$ & $P$ \\
\hline Age $>60 y$ & $1.51(0.82-2.77)$ & 0.18 & $0.96(0.60-1.54)$ & 0.86 \\
\hline Ann Arbor stage III/IV & $1.16(0.54-2.50)$ & 0.70 & $1.21(0.70-2.08)$ & 0.50 \\
\hline$>1$ extranodal site & $1.27(0.66-2.46)$ & 0.47 & $1.20(0.73-1.96)$ & 0.47 \\
\hline LDH level $>$ normal & $2.42(1.26-4.67)$ & 0.01 & $2.22(1.39-3.56)$ & $<0.01$ \\
\hline Eastern Cooperative Oncology Group performance status $\geq 2$ & $1.55(0.79-3.05)$ & 0.21 & $1.56(0.93-2.64)$ & 0.10 \\
\hline PET-/BMB + & $0.49(0.07-3.68)$ & 0.49 & $1.13(0.40-3.20)$ & 0.83 \\
\hline PET+/BMB- & $0.74(0.33-1.66)$ & 0.46 & $0.68(0.37-1.27)$ & 0.23 \\
\hline $\mathrm{PET}+/ \mathrm{BMB}+$ & $2.26(1.02-5.03)$ & 0.05 & $1.61(0.84-3.10)$ & 0.15 \\
\hline
\end{tabular}

Data in parentheses are $95 \%$ Cls. 
hematopoiesis $(11,20)$. There is evidence from histopathologic studies that a DLBCL infiltrate of $10 \%$ cells in iliac crest marrow does not impact survival (21), and indolent lymphoma in the marrow of patients with DLBCL does not influence progression risk independent of the IPI (4). It is therefore to be expected that PET-/BMB + cases in this cohort had survivals that were not inferior to cases without identified marrow disease.

It is well recognized that diffuse ${ }^{18} \mathrm{~F}-\mathrm{FDG}$ uptake in marrow may be increased above background due to reactive myelopoiesis in both Hodgkin and non-Hodgkin lymphoma $(6,22)$. Differentiation of reactive from pathologic diffuse PET positivity due to lymphoma may improve with experience (11). This study confirms that cases with diffuse ${ }^{18} \mathrm{~F}-\mathrm{FDG}$ accumulation above background in marrow should always be biopsied to establish etiology.

Hence, data from our large cohort add support to the limited existing data that a positive marrow biopsy does not add useful information for treatment planning or outcome prediction when the marrow is negative on staging PET (13). However, the experience from this international cohort leads us to disagree with the conclusion of Adams et al. (13) that diffuse bone marrow ${ }^{18} \mathrm{~F}$-FDG uptake throughout the skeletal marrow should be regarded as marrow involvement, without the need for corroboration by biopsy.

The prognostic impact of focal ${ }^{18} \mathrm{~F}$-FDG uptake in marrow has, until now, remained unclear. Reported series to date have had insufficient numbers to analyze the outcome of this group separately and have reached opposing conclusions as to whether focal PET disease on its own is an independent adverse prognostic indicator (10-12). With this larger cohort we have, for the first time to our knowledge, been able to demonstrate that cases with focal ${ }^{18} \mathrm{~F}$ FDG-avid deposits on PET, without positive histology, have EFSs and OSs that do not differ significantly from DLBCL cases without spread to marrow (EFS, $78 \%$, vs. $81 \%$, respectively). Focal PET-positive cases with positive histology had significantly inferior survival (EFS, 46\%) (Fig. 3). The likely explanation is that the probability of an untargeted biopsy sampling a metastatic lymphoma deposit increases with the extent of marrow disease. Hence, it is this, rather than marrow involvement per se, that confers poor prognosis.

Our conclusion fits with that of Khan et al. who noted that PET/CT had high sensitivity for limited marrow diseases, whereas biopsy detects only the most extensive marrow involvement (11). Their conclusion was corroborated by finding that a positive marrow biopsy was strongly associated with spread to 4 or more extranodal organ sites. As the present study recorded only 1 - versus 2 -or-more-organ involvement, we were unable to provide confirmation that extensive extranodal disease on staging PET obviated the need for a marrow biopsy.

The unique international nature of this prospective, protocoldriven study brings with it both strengths and weaknesses. Geographic separation prohibited the sharing of histologic material for central review. PET scans were read centrally for treatment response but not consistently for bone marrow involvement. Less experienced centers appeared to overinterpret diffuse ${ }^{18} \mathrm{~F}-\mathrm{FDG}$ uptake in marrow as lymphoma infiltration rather than reactive myelopoiesis. Nonetheless, our results have the unusual strength of being internationally applicable.

The new data from this large cohort provide a clearer answer to the question whether to use bone marrow staging by biopsy or PET for defining prognosis in DLBCL (23). The answer appears to be that both are important but not necessary for every patient. The patient with normal marrow on staging PET is unlikely to gain prognostic information from a routine marrow biopsy. However, when the marrow is abnormal on staging PET, the presence or absence of lymphoma in iliac crest histology has an important impact on outcome. When this algorithm is used, only a quarter of patients might need a biopsy.

\section{CONCLUSION}

We have demonstrated that neither bone marrow histology nor PET alone is a reliable indicator of poor risk marrow disease. The most consistent indicator is marrow involvement identified by both PET and histology. We suggest that the algorithm we propose above should be incorporated into practice guidelines and would strengthen the new IPI (3). The strategy we outline would reduce the burden on patients and improve the precise identification of poor prognosis DLBCL internationally.

\section{DISCLOSURE}

The costs of publication of this article were defrayed in part by the payment of page charges. Therefore, and solely to indicate this fact, this article is hereby marked "advertisement" in accordance with 18 USC section 1734. The IAEA funded three collaborator meetings and a writing group meeting. Some contribution was made to each participating center to partially defray the cost of additional study PET scans. The IAEA did not influence analysis or interpretation of the data or the decision to publish. No other potential conflict of interest relevant to this article was reported.

\section{ACKNOWLEDGMENTS}

Particular thanks go to Dr. Baldip Khan and Professor Maurizio Dondi for initiating and supporting the project under the umbrella of the International Atomic Energy Agency.

\section{REFERENCES}

1. The International Non-Hodgkin's Lymphoma Prognostic Factors Project. A predictive model for aggressive non-Hodgkin's lymphoma. N Engl J Med. 1993;329:987-994.

2. Sehn LH, Berry B, Chhanabhai M, et al. The revised International Prognositic Index (R-IPI) is a better predictor of outcome than the standard IPI for patients with diffuse large B-cell lymphoma treated with R-CHOP. Blood. 2007;109:18571861.

3. Zhou Z, Sehn L, Rademaker AW, et al. An enhanced International Prognostic Index (NCCN-IPI) for patients with diffuse large B-cell lymphoma treated in the rituximab era. Blood. 2014;123:837-842.

4. Sehn LH, Scott WS, Chhanabhai M, et al. Impact of concordant and discordant bone marrow involvement on outcome in diffuse large B-cell lymphoma treated with R-CHOP. J Clin Oncol. 2011;29:1452-1457.

5. Partridge S, Timothy A, O'Doherty M, et al. 2-fluorine-18-fluoro-2-deoxy-D glucose positron emission tomography in the pretreatment staging of Hodgkin's disease: influence on patient management in a single institution. Ann Oncol. 2000;11:1273-1279.

6. Carr R, Barrington S, Madan B, et al. Detection of lymphoma in bone marrow by whole-body positron emission tomography. Blood. 1998;91:3340-3346.

7. Pakos EE, Fotopoulos AD, Ioannidis JP. ${ }^{18} \mathrm{~F}-\mathrm{FDG}$ PET for evaluation of bone marrow infiltration in staging of lymphoma: a meta-analysis. J Nucl Med. 2005;46:958-963.

8. Pelosi E, Penna D, Douroukas A, et al. Bone marrow disease detection with FDG-PET/CT and bone marrow biopsy during the staging of malignant lymphoma: results from a large multicentre study. $Q \mathrm{~J} \mathrm{Nucl} \mathrm{Med} \mathrm{Mol} \mathrm{Im-}$ aging. 2011;55:469-475.

9. Chen YK, Yeh CL, Tsui CC, Liang J-A, Chen J-H, Kao C-H. F-18 FDG PET for evaluation of bone marrow involvement in non-Hodgkin lymphoma: a metaanalysis. Clin Nucl Med. 2011;36:553-559.

10. Hong J, Lee Y, Kim G, et al. Role of FDG-PET/CT in detecting lymphomatous bone marrow involvement in patients with newly diagnosed diffuse large B-cell lymphoma. Ann Hematol. 2012;91:687-695. 
11. Khan AB, Barrington S, Mikhaeel NG, et al. PET-CT staging of DLBCL accurately identifies and provides new insight into the clinical significance of bone marrow involvement. Blood. 2013;122:61-67.

12. Berthet L, Cochet A, Kanoun S, et al. In newly diagnosed diffuse large B-cell lymphoma, determination of bone marrow involvement with ${ }^{18} \mathrm{~F}$-FDG PET-CT provided better diagnostic performance and prognostic stratification than does biopsy. J Nucl Med. 2013;54:1244-1250.

13. Adams HJA, Kwee TC, de Keizer B, Fijnheer R, de Klerk LMH, Nievelstein RAJ. FDG PET/CT for the detection of bone marrow involvement in diffuse large B-cell lymphoma: systematic review and meta-analysis. Eur J Nucl Med Mol Imaging. 2014;41:565-574.

14. Barrrington SF, Mikhaeel NG, Kostakoglu L, et al. Role of imaging in the staging and response assessment of lymphoma: consensus of the International Conference on Malignant Lymphomas Imaging Working Group. J Clin Oncol. In press.

15. Swerdlow SH, Campo E, Harris NL, et al. World Health Organization Classification of Tumours of Haematopoietic and Lymphoid Tissues. Lyon, France: IARC; 2008.

16. El-Galaly TC, d'Amore F, Mylam KJ, et al. Routine bone marrow biopsy has little or no therapeutic consequences for positron emission tomography/computed tomography-staged treatment-naïve patients with Hodgkin Lymphoma. J Clin Oncol. 2012;30:4508-4514.
17. Cheson BD, Pfistner B, Juweid RD, et al. Revised response criteria for malignant lymphoma. J Clin Oncol. 2007;25:579-586.

18. Hrynaszkiewicz I, Norton ML, Vickers AJ, Altman DG. Preparing raw clinical data for publication: guidance for journal editors, authors, and peer to the question, bone marrow staging reviewers. BMJ. 2010;340:c181.

19. Cox DR. Regression models and life tables. J Royal Statistical Soc. 1972, series B. 1972;34:187-220.

20. Paone G, Itti E, Haioun C, et al. Bone marrow involvement in diffuse large B-cell lymphoma: correlation between FDG-PET uptake and type of cellular infiltrate. Eur J Nucl Med Mol Imaging. 2009;36:745-750.

21. Campbell J, Seymour JF, Matthews J, Wolf M, Stone J, Juneja S. The prognostic impact of bone marrow involvement in patients with diffuse large cell lymphoma varies according to the degree of infiltration and presence of discordant marrow involvement. Eur J Haematol. 2006;76:473-480.

22. Elstrom RL, Tsai DE, Vergillio JA, Downs LH, Alavi A, Schuster SJ. Enhanced marrow $\left[{ }^{18} \mathrm{~F}\right]$ fluorodeoxyglucose uptake related to myeloid hyperplasia in Hodgkin's lymphoma can simulate lymphoma involvement in marrow. Clin Lymphoma. 2004;5:62-64.

23. Avigdor A. Staging of DLBCL: bone marrow biopsy or PET-CT? Blood. $2013 ; 122: 4-5$. 